\title{
Ultrahigh Resolution Scatterometer Winds near Hawaii
}

\author{
Nolan Hutchings ${ }^{1,+}+\mathbb{C}$, Thomas Kilpatrick ${ }^{2}$ and David G. Long $1, t, * \mathbb{C}$ \\ 1 Department of Electrical \& Computer Engineering, Brigham Young University, 450 EB, Provo, UT 84602, \\ USA; nolanhutchings63@gmail.com \\ 2 Scripps Institution of Oceanography, University of California San Diego, 9500 Gilman Dr MC 0206, La Jolla, \\ CA 92093, USA; tkilpatrick@ucsd.edu \\ * Correspondence: long@byu.edu \\ + These authors contributed equally to this work.
}

Received: 8 January 2020; Accepted: 5 February 2020; Published: 8 February 2020

check for updates

\begin{abstract}
Hawaii regional climate model (HRCM), QuikSCAT, and ASCAT wind estimates are compared in the lee of Hawaii's Big Island with the goal of understanding ultrahigh resolution (UHR) scatterometer wind retrieval capabilities in this area, which includes a reverse-flow toward the island in the lee of the predominate flow. A comparison of scatterometer measured $\sigma^{0}$ and model predicted $\sigma^{0}$ suggests that scatterometers can detect the reverse flow in the lee of the island; however, neither QuikSCAT- nor ASCAT-estimated winds consistently report this flow. Furthermore, the scatterometer UHR winds do not resolve the wind direction features predicted by the HRCM. Differences between scatterometer measured $\sigma^{0}$ and HRCM predicted $\sigma^{0}$ indicate possible error in the placement of key reverse flow features predicted by the HRCM. We find that coarse initialization fields and a large size median filter windows used in ambiguity selection can impede the accuracy of the UHR wind direction retrieval in this area, suggesting the need for further development of improved near-coastal ambiguity selection algorithms.
\end{abstract}

Keywords: scatterometer; ocean winds; wind retrieval

\section{Introduction}

Satellite radar instruments called wind scatterometers illuminate the Earth's surface with microwaves and measure the normalized radar cross-section $\left(\sigma^{0}\right)$ of the surface [1]. While the $\sigma^{0}$ measurements prove useful for many applications such as iceberg tracking, ice classification, vegetation classification, and soil moisture estimation [1-4], the scatterometer's primary function is to estimate the near-surface vector wind from the ocean $\sigma^{0}$ measurements.

From $\sigma^{0}$ measured over the ocean, estimates of wind speed and direction can be made over the open ocean by combining $\sigma^{0}$ measurements taken from different azimuth angles [1]. Unlike wind measurements from buoys, ships, and planes, scatterometers provide regular global wind vector estimates over large regions of open ocean. The large coverage and availability of scatterometer data proves useful for weather studies and prediction $[1,5,6]$. Traditional wind estimates are retrieved on a coarse $12.5 \mathrm{~km}$ or $25 \mathrm{~km}$ grid (referred to as L2B estimates) and are excellent for understanding large scale wind flow and other features. Wind estimates close to shore are discarded due to land contamination of ocean $\sigma^{0}$ [7].

Ocean $\sigma^{0}$ are related to wind speed and direction through a geophysical model function (GMF) $[1,8,9]$. In the GMF, a single $\sigma^{0}$ value corresponds to multiple wind speed and direction pairs. Hence, multiple $\sigma^{0}$ observed at different look angles are needed to narrow the solution space [10]. Even with multiple $\sigma^{0}$, this process results in two to four ambiguous wind vector solutions for each 
resolution element (wind vector cell, WVC) [8]. From the ambiguous wind vector solutions, a final wind field is chosen. This process of "ambiguity selection" begins by looking to an outside data set, typically coarser in resolution, to initialize the ambiguity choices [10]. The initialization field, sometimes referred to as a "nudging field", ensures that general wind trends are present.

After nudging, the wind ambiguities are further processed using a median filter based ambiguity selection scheme $[8,11,12]$. The median filter based ambiguity selection scheme is not a true median filter because it uses only the values available in the ambiguities. The median filter selection preserves wind fronts and reduces noise, but as shown later, is limited by the size of the median filter window.

With their high topography, the Hawaiian Islands greatly affect nearby wind conditions. Orographic wind forcing near Hawaii not only influences local conditions, but extends far across the Pacific Ocean $[13,14]$. Accurately observing and modeling these orographic winds is important in understanding wind forcing of ocean circulation. Furthermore, improvements in Ultrahigh resolution (UHR) wind retrieval can benefit other near-coastal areas with orographic wind forcing conditions.

Wind estimation near the Hawaiian islands is a challenge for L2B coarse resolution estimates. Complex fine resolution wind direction features and low wind speeds lead to systematic errors in L2B wind estimation in the lee of Hawaii's Big Island for both QuikSCAT and ASCAT [15]. Wind directions in the lee of the Big Island can run counter to the prevailing trade winds, exhibiting a reverse flow toward the island. The reverse flow has been both observed [16-18] and modeled [19,20]. The reverse flow can be seen in the University of Hawaii's numerical model winds [21]. The model is referred to as the Hawaii regional climate model (HRCM), which provides predicted vector winds hourly on a $3 \mathrm{~km}$ grid near the Hawaiian islands [19-22]. The reverse flow is only rarely observed in L2B winds [15].

Figure 1 illustrates an HRCM wind field from a summer day and shows key wind features expected in this area. Figure 1 shows trade winds blowing around the two peaks of the Big Island creating regions of high wind speed to the north and south of the island and a low wind speed tail in the lee (west side) of the island. The low wind speed tail is where the reverse flow is found. Due to the consistency of the trade winds over Hawaii, generally, as in Figure 1, the reverse flow is found on the west side of the island. However, the reverse flow phenomenon can be observed on other sides of the island, depending on prevailing wind. The angle and length of the reverse flow region depends on the direction and speed of the prevailing wind flow. The reverse flow region can extend as far out as 50-100 km from the shore $[15,16]$.

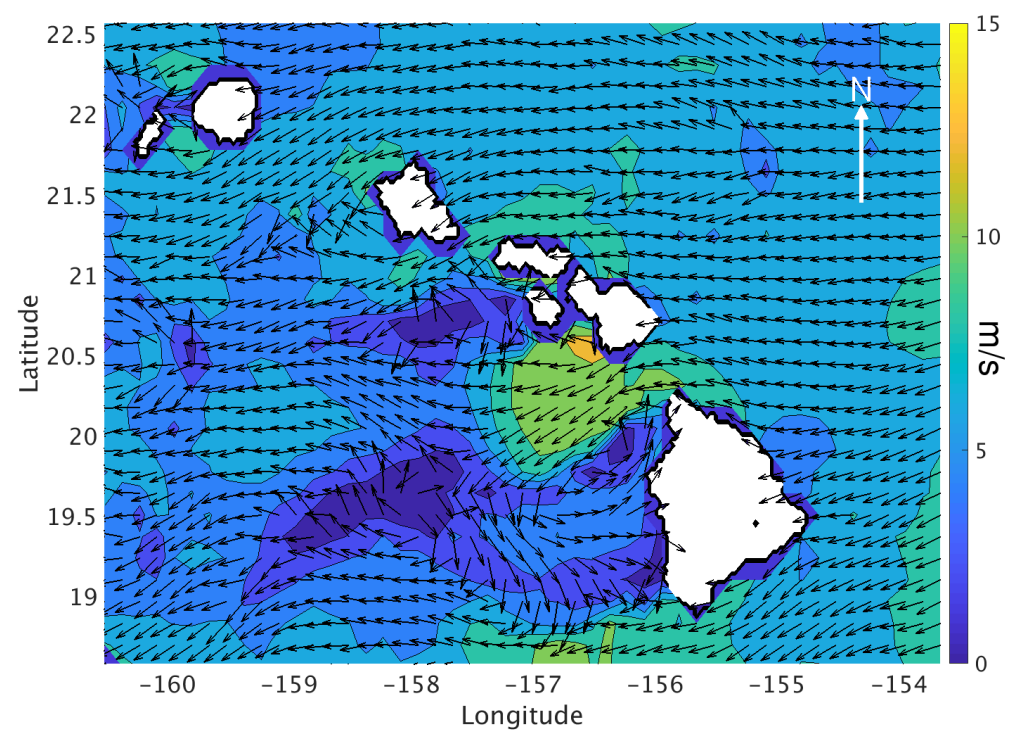

Figure 1. An example HRCM 3 km hourly wind vector field from 3:00 a.m. 26 June 2003. Wind speed is shown in color and wind direction quivers are downsampled and unit length. The land mask is shown in white. The reverse flow can be seen on the west side of the Big Island. 
In the case of Figure 1, the model shows a reverse flow resulting from the formation of one vortex. Often, multiple vortices (counter clockwise and/or clockwise) contribute to the reverse flow. The locations and intensities of the vortices vary with changing wind flow.

Resolution enhancement and reconstruction techniques enable estimation of winds on an ultrahigh resolution (UHR) $2.5 \mathrm{~km}$ or $1.25 \mathrm{~km}$ grid [23-25]. UHR products have been produced for QuikSCAT [25-29], RapidSCAT [30], and ASCAT [31,32]. UHR wind processing reveals finer resolution detail of ocean wind phenomena and gives valuable insight to close to shore wind phenomena. Finer resolution wind products allow wind estimation closer to shore through land contamination removal (LCR) $[7,32]$.

This paper explores the UHR capabilities of QuikSCAT at $2.5 \mathrm{~km}$ and ASCAT at $1.25 \mathrm{~km}$ to resolve the complex wind features in the lee of the Hawaii's Big Island. In Section 2, a comparison between QuikSCAT and ASCAT UHR winds and HRCM winds is outlined. In Section 3, scatterometer measured $\sigma^{0}$ are compared to model predicted $\sigma^{0}$. Section 4 shows the effects of the nudging field and median filter-based ambiguity selection scheme on UHR scatterometer wind fields. Conclusions are discussed in Section 5.

\section{Scatterometer UHR Wind Estimates in the Lee of the Big Island}

In this paper, QuikSCAT and ASCAT passes from January 1, 2007 to December 31, 2008 are considered for comparison with HRCM winds. The region of interest is between $20.50^{\circ}$ and $18.75^{\circ}$ latitude and $-154^{\circ}$ and $-158^{\circ}$ longitude around the Big Island. The high variability of winds in the lee of the Big Island necessitates a close temporal collocation. In this analysis, only QuikSCAT passes within 10 minutes and ASCAT passes within 20 minutes of the HRCM wind fields are used. ASCAT orbits have a broader collocation window because only a few orbits are within 10 minutes of the HRCM winds. For consistency, only scatterometer orbits with mean trade wind flow of around $270^{\circ}$ that puts the reverse flow on the west side of the island are considered. From those years, a total of 276 QuikSCAT and 236 ASCAT passes are studied.

We do not expect a perfect correspondence between the QuikSCAT and ASCAT observations and HRCM winds because the HRCM winds are constrained only by observations at the lateral boundaries. Thus, spontaneous behavior in the lee vortices and reverse flow shown by the model likely may not match the scatterometer observations. Nevertheless, the HRCM winds are a useful tool for comparison. It is important to note that HRCM winds predict wind speeds well below $5 \mathrm{~m} / \mathrm{s}$, a range where scatterometers are less accurate due to lower SNR [33]. Scatterometer winds below $2 \mathrm{~m} / \mathrm{s}$ are considered unreliable.

HRCM provides winds near and over the islands; however, scatterometer wind retrieval is limited to the ocean sufficiently far from land. For this reason, comparisons with UHR winds consider only HRCM winds outside of the scatterometer ocean near-coastal zone land contamination buffer. In the following, QuikSCAT UHR wind speeds and directions are first compared with the HRCM winds. Then, a comparison of ASCAT UHR wind vectors with HRCM winds concludes. While we do compare the wind speeds, the focus of the analysis is wind directions.

Figure 2a compares collocated QuikSCAT UHR and HRCM wind speeds (all collocations are within $2.5 \mathrm{~km}$ ). The wind speeds between the two compare well. We note that the global mean wind speed over the open ocean is approximately $7 \mathrm{~m} / \mathrm{s}$. At low wind speeds, QuikSCAT wind speed estimates are biased somewhat higher compared to the model predicted speeds. Figure $2 \mathrm{~b}$ compares QuikSCAT UHR wind directions with HRCM wind directions. From the figure, it is apparent that the trade winds (around $270^{\circ}$ ) dominate the UHR wind direction field even when HRCM suggests directions other than the trade winds. QuikSCAT UHR winds do not show the reverse flow or vortex features that are included in the HRCM winds. 


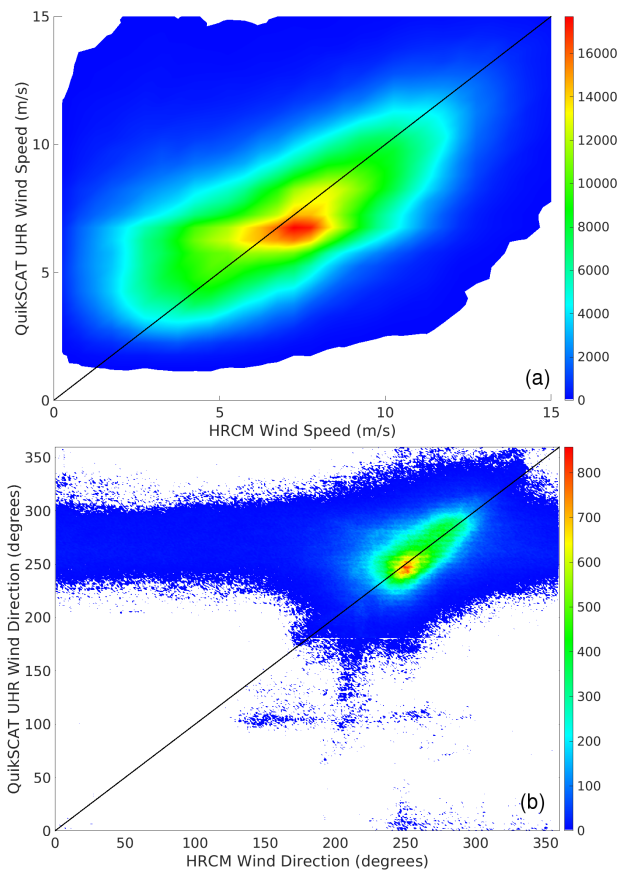

Figure 2. Density plots of QuikSCAT UHR wind speeds (a) and directions (b) plotted versus HRCM winds. A $y=x$ line is included in each plot for reference.

Figure 3 compares ASCAT UHR wind speeds and directions to HRCM winds (all collocations are within $1.25 \mathrm{~km}$ ). ASCAT UHR and HRCM wind speeds show a similar distribution to that shown in Figure 2, though, for these particular ASCAT passes, the mean observed wind speed is somewhat higher than the mean QuikSCAT winds. Figure 3 shows that ASCAT wind directions are also dominated by trade wind flow and do not resolve the reverse flow and wind direction features of the HRCM winds.
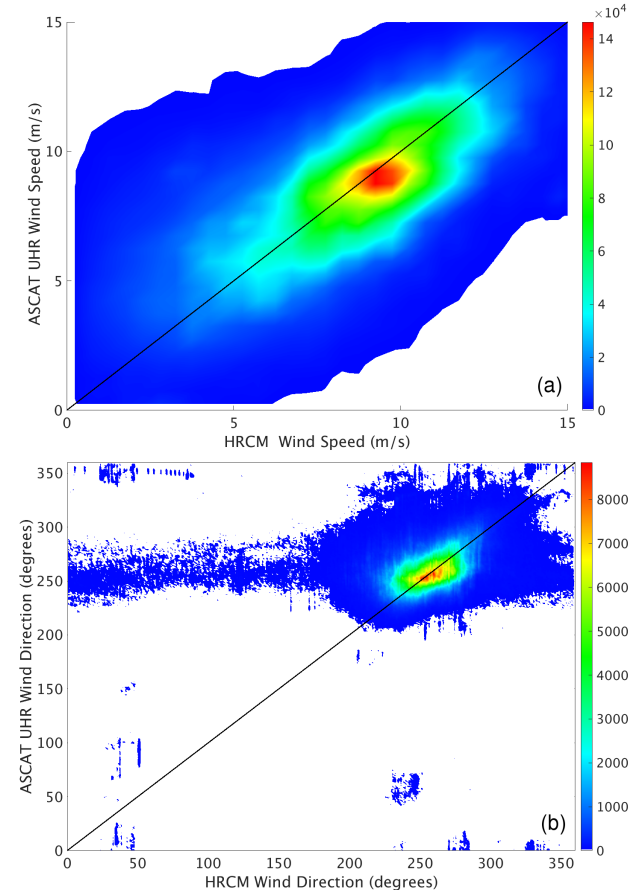

Figure 3. ASCAT UHR wind speeds (a) and directions (b) collocated with HRCM winds. A $y=x$ line is included in each plot for reference. 
In both cases, the scatterometer wind directions do not match well what the HRCM winds predict. In particular, scatterometer winds do not show the reverse flow and vortices seen in the HRCM winds. While the HRCM winds do show reverse flow and vortices, the true locations of any vortices and reverse flow cannot be verified. With no truth data, it is difficult to draw too many conclusions from the above analysis. These results do show, however, that the reverse flow and vortices are not resolved in current UHR wind retrieval for QuikSCAT and ASCAT. In the next section, we turn to an analysis of $\sigma^{0}$ to further compare UHR products with the HRCM.

\section{Comparison of Scatterometer Measured $\sigma^{0}$ and Model Predicted $\sigma^{0}$}

Scatterometer measured $\sigma^{0}$ are useful to examine because they are directly related to wind stress [34]. The measurements are noisy, but they are unaffected by imperfect wind retrieval and ambiguity selection routines. In this section, scatterometer measured $\sigma^{0}$ are compared with predicted $\sigma^{0}$ from HRCM and European Centre for Medium-Range Weather Forecasts numerical weather prediction (NWP) winds. The predicted $\sigma^{0}$ values are generated for each model wind set using the the QuikSCAT QMOD3 and ASCAT CMOD5 GMFs [8,9].

Comparison between the measured $\sigma^{0}$ and HRCM predicted $\sigma^{0}$ can show if the scatterometers' $\sigma^{0}$ measurements are possibly detecting the reverse flow and vortices. The coarse NWP wind fields show only the trade wind flow and are a good baseline comparison to see if scatterometer measured $\sigma^{0}$ are detecting disturbances in the trade wind flow. The area of interest for this study is the same as in the previous section. For consistency, only scatterometer passes with mean trade wind flow of around $270^{\circ}$ that puts the reverse flow on the west side of the island are considered. The same temporal collocation criteria are used in this comparison as in Section 2. However, additional stipulations are applied to the scatterometer passes considered for this analysis.

QuikSCAT's rotating pencil beam results in changing antenna observation angles across the swath. Thus, only QuikSCAT passes with similar antenna look angles are considered. Similarly, ASCAT's left and right swaths have sufficiently different azimuth angles and are compared separately. Furthermore, the incidence angle of fan beam scatterometers changes across the swath for each beam so passes over Hawaii should be compared with like incidence angles. Only the left swath measurements and passes with similar incidence angles over Hawaii are used in this study. This results in a total of 104 QuikSCAT orbits and 44 ASCAT orbits for analysis. First, QuikSCAT is compared to HRCM and NWP predicted $\sigma^{0}$ in Section 3.1; a comparison between ASCAT $\sigma^{0}$ and predicted $\sigma^{0}$ from the models follows in Section 3.2.

\subsection{QuikSCAT versus Model Predicted $\sigma^{0}$}

The average difference between the QuikSCAT measured $\sigma^{0}$ and HRCM QMOD3 predicted $\sigma^{0}$ for each "flavor" of $\sigma^{0}$ is shown in Figure $4 \mathrm{a}-\mathrm{d}$. The four flavors of $\sigma^{0}$ for pencil beam scatterometers are vertical polarized fore looking (VF), vertical polarized aft looking (VA), horizontal polarized fore looking (HF), and horizontal polarized aft looking (HA). The flavors are analyzed separately because of the different effects azimuth angle and polarization have on $\sigma^{0}$. The first row in Figure 4 shows VF, the second VA, the third HF, and the fourth row shows HA. 

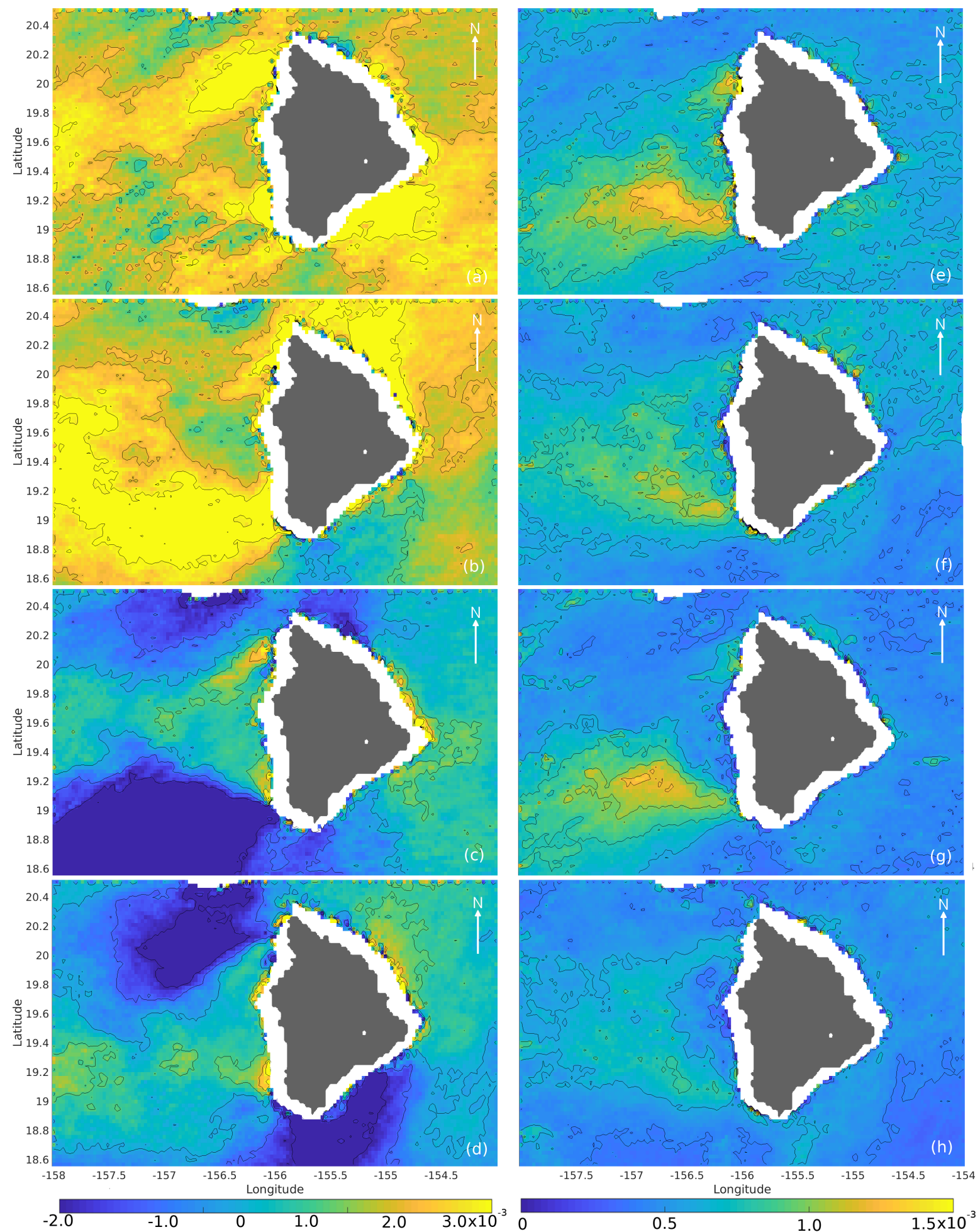

Figure 4. Panels (a-d) show the average difference in linear values between measured QuikSCAT $\sigma^{0}$ and HRCM predicted $\sigma^{0}$ for each flavor of $\sigma^{0} ;(\mathbf{e}-\mathbf{h})$ show the standard deviation of the difference between measured QuikSCAT $\sigma^{0}$ and HRCM predicted $\sigma^{0}$ normalized by QuikSCAT average wind speeds in linear values. The first row shows values for VF, the second is VA, the third HF, and the fourth row is HA. Land is shown in gray and the land contamination buffer in white.

Despite the differences in the high wind speed regions, there is a low mean difference immediately west of the Big Island seen in all polarizations. This is expected because, even if the two data sets have different orientations for the low wind speed tail, the part immediately behind the island is common 
between them. However, the low difference is interesting because the QuikSCAT ambiguity-selected winds in this area are generally $180^{\circ}$ opposite of the HRCM directions. The $\sigma^{0}$ agreeing in this area suggests that QuikSCAT $\sigma^{0}$ may be detecting the reverse flow, even if the selected QuikSCAT wind directions do not match the model winds there. The differences in wind speed and direction between the two may be because the wind ambiguity selection is in error in the scatterometer winds. It is important to note that the wake of the island is a low speed region, so we do expect a lower difference in this area due to lower $\sigma^{0}$ values since $\sigma^{0}$ is directly proportional to a power of the wind speed.

Figure $4 \mathrm{e}-\mathrm{h}$ shows the standard deviation of the difference between the $\sigma^{0}$ normalized by QuikSCAT average wind speeds. We normalize with respect to QuikSCAT average wind speeds because $\sigma^{0}$ values are more variable as wind speed increases. This normalization has the effect of lowering the standard deviation values in high wind speed areas. This partially removes the effect of wind speed on the comparison.

In Figure $4 \mathrm{e}-\mathrm{h}$, the standard deviation is the highest in the southern high wind speed area. Variation in the differences in the high wind speed area suggests that HRCM's predicted wind tail and reverse flow features do not match what QuikSCAT is detecting. The low standard deviation directly west of the island indicates that both wind fields are consistently close in that area while the low difference in $\sigma^{0}$ and low standard deviation values suggest that QuikSCAT $\sigma^{0}$ is detecting a reverse flow.

The comparisons between the two data sets are clearly dependent on azimuth angle as seen by the "mirroring" of the differences in the north and south high wind speed areas between the fore and aft observations (compare Figure $4 \mathrm{a}$ and $\mathrm{b}$ or $\mathrm{c}$ and $\mathrm{d}$ ). The effect of azimuth angle on $\sigma^{0}$ is explained below with some ASCAT measurements near the Big Island. We show this with ASCAT measurements and NWP predicted $\sigma^{0}$ because of how clearly they illustrate the effect of azimuth angle.

$\sigma^{0}$ is a function of wind speed and $\chi$ (difference between antenna look angle and wind direction). As wind speed increases so does the variability of $\sigma^{0}$ with respect to $\chi$. This can be seen in Figure $5 \mathrm{a}$ in the CMOD5 $\sigma^{0}$ curves plotted at different wind speeds. At $5 \mathrm{~m} / \mathrm{s}, \sigma^{0}$ varies little as $\chi$ changes. In contrast, at $15 \mathrm{~m} / \mathrm{s}$, the $\sigma^{0}$ span a much larger range of values as $\chi$ changes.

In Figure $5 \mathrm{a}-\mathrm{c}$, ASCAT $\sigma^{0}$ values at a point in the north (red) and in the south (blue) high wind speed areas are plotted versus $\chi$. The ASCAT selected wind speeds and directions of the north and south locations are similar to each other, but the $\sigma^{0}$ at those locations vary slightly due to $\chi$.

Figure $5 \mathrm{~d}-\mathrm{f}$ show NWP predicted $\sigma^{0}$ taken from the same north and south locations as the ASCAT measurements. The NWP wind speeds and directions at the north and south locations are similar to each other. In general, the reported NWP wind speeds are much lower than that of ASCAT and fall on a $\sigma^{0}$ curve that does not vary much with $\chi$. Comparing a and $\mathrm{d}$ shows that there is a greater difference between the ASCAT measured $\sigma^{0}$ and NWP predicted $\sigma^{0}$ at the north end. In contrast, the $\sigma^{0}$ at the southern end have a relatively small difference. A similar comparison of a different beam in c and $\mathrm{f}$ shows that there is a greater difference between $\sigma^{0}$ at the southern end and the difference at the northern end is relatively small. In the mid antenna look (b and $\mathbf{e})$, both north and south points have similar differences in $\sigma^{0}$.

The change in differences in $\sigma^{0}$ is explained by a change in antenna azimuth look angle which affects $\chi$. The ASCAT and NWP wind speeds and directions are different but how well that shows up in the $\sigma^{0}$ depends on the look angle. This effect can be seen to varying degrees in Figure 4 and other figures in this section. The high variability of HRCM winds makes it difficult to precisely identify this effect, but artifacts can still be seen. 

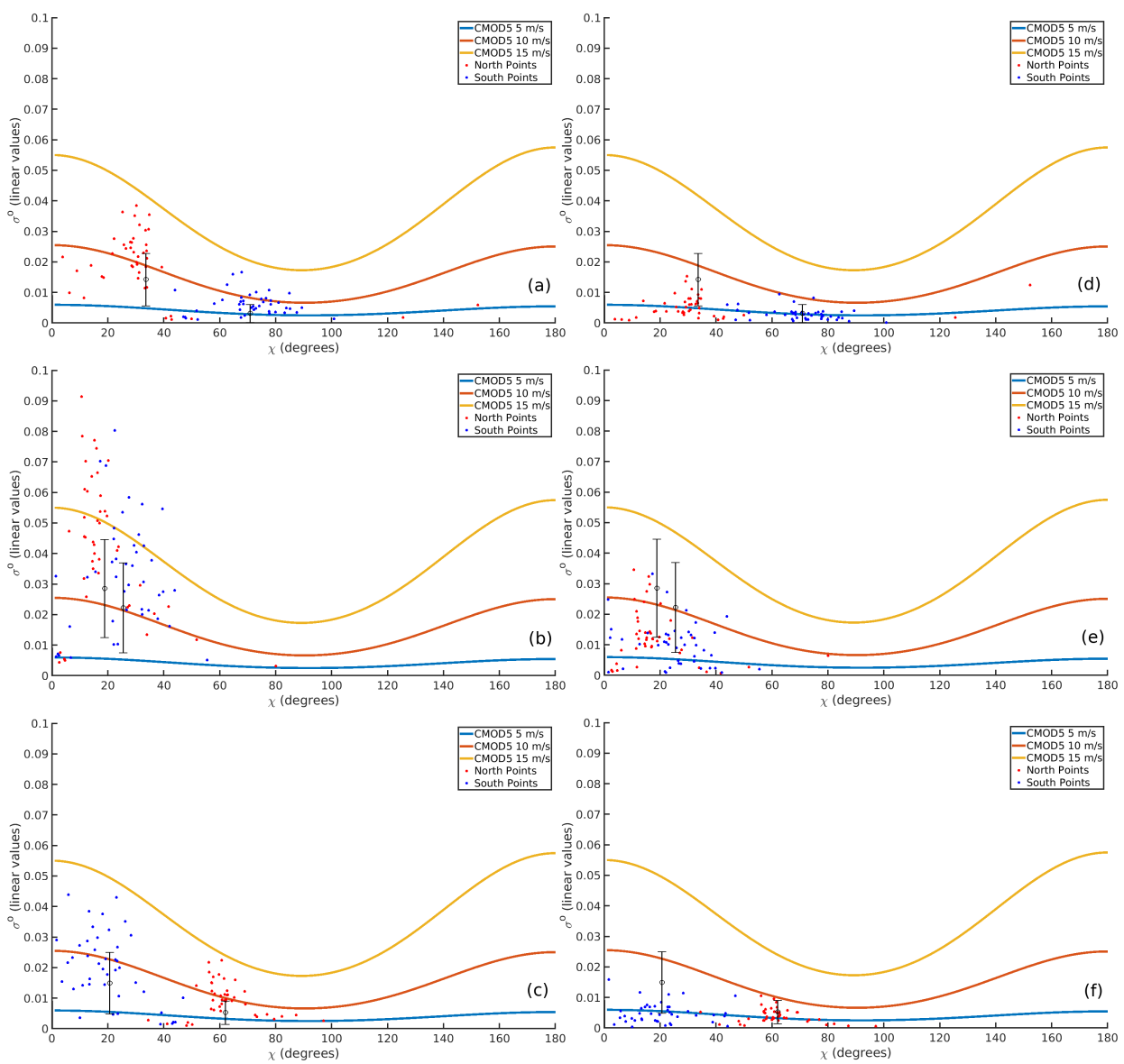

Figure 5. ASCAT $\sigma^{0}$ from multiple revs from a point (see Figure 8 a) in the north high wind speed region and a point in the south high wind speed region are plotted for fore (a), mid (b), and aft (c) looks. Corresponding NWP predicted $\sigma^{0}$ are shown in (d-f) for the same beams. $\sigma^{0}$ curves at $50^{\circ}$ incidence angle for CMOD5 for 5, 10, and $15 \mathrm{~m} / \mathrm{s}$ are plotted in each panel for reference. Error bars showing the average difference between ASCAT measured $\sigma^{0}$ and NWP predicted $\sigma^{0}$ and the standard deviation of the differences are plotted in black.

Measured QuikSCAT $\sigma^{0}$ are compared with coarse NWP predicted $\sigma^{0}$. Figure 6a-d shows the average difference between measured QuikSCAT $\sigma^{0}$ and QMOD3 NWP predicted $\sigma^{0}$. As expected, the average differences show that NWP is not modeling what QuikSCAT is detecting. There are large differences in the high wind speed regions, especially for the vertical polarization. Both polarizations have a low average difference immediately west of the Big Island. The horizontal polarization shows differences in the same areas but with smaller differences on average. This is consistent with the the QuikSCAT horizontal polarization being lower than the HRCM $\sigma^{0}$ seen in Figure $4 \mathrm{c}$, d.

Figure 6e-h show the standard deviation of the differences for QuikSCAT and NWP $\sigma^{0}$ normalized by QuikSCAT average wind speeds. There are high standard deviation values (especially for vertical polarization) west of the island even in the area immediately behind the island where a reverse flow is expected. The disagreement between NWP predicted $\sigma^{0}$ (which represent only the trade wind flow) and QuikSCAT $\sigma^{0}$ measurements further suggests that QuikSCAT $\sigma^{0}$ are possibly detecting a reverse flow and vortices. 

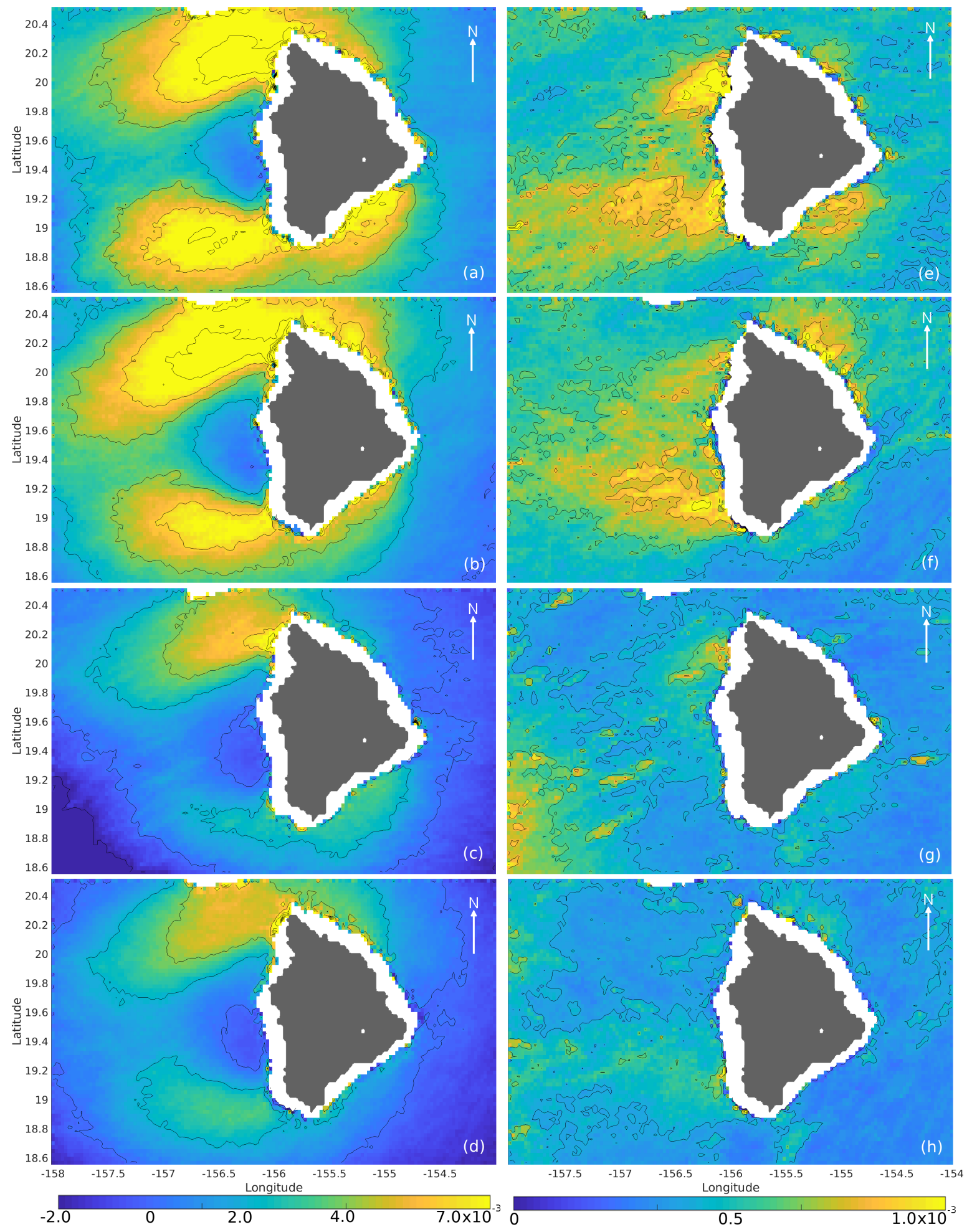

Figure 6. Panels (a-d) show the average difference in linear values between measured QuikSCAT $\sigma^{0}$ and NWP predicted $\sigma^{0}$ for different flavors of $\sigma^{0} ;(\mathbf{e}-\mathbf{h})$ show the normalized standard deviation in linear values between measured QuikSCAT $\sigma^{0}$ and NWP predicted $\sigma^{0}$. The first row is VF, second is VA, third HF, and the fourth row HA. The land is shown in gray and the land contamination buffer is shown in white. 


\subsection{ASCAT versus Model Predicted $\sigma^{0}$}

The average difference between the ASCAT measured $\sigma^{0}$ and HRCM CMOD5 predicted $\sigma^{0}$ is shown in Figure 7. The near vertical boundary feature directly right of the island is an artifact resulting from the swath edge in different passes where there is limited coverage from ASCAT passes. Data from that area right of the island can be ignored.

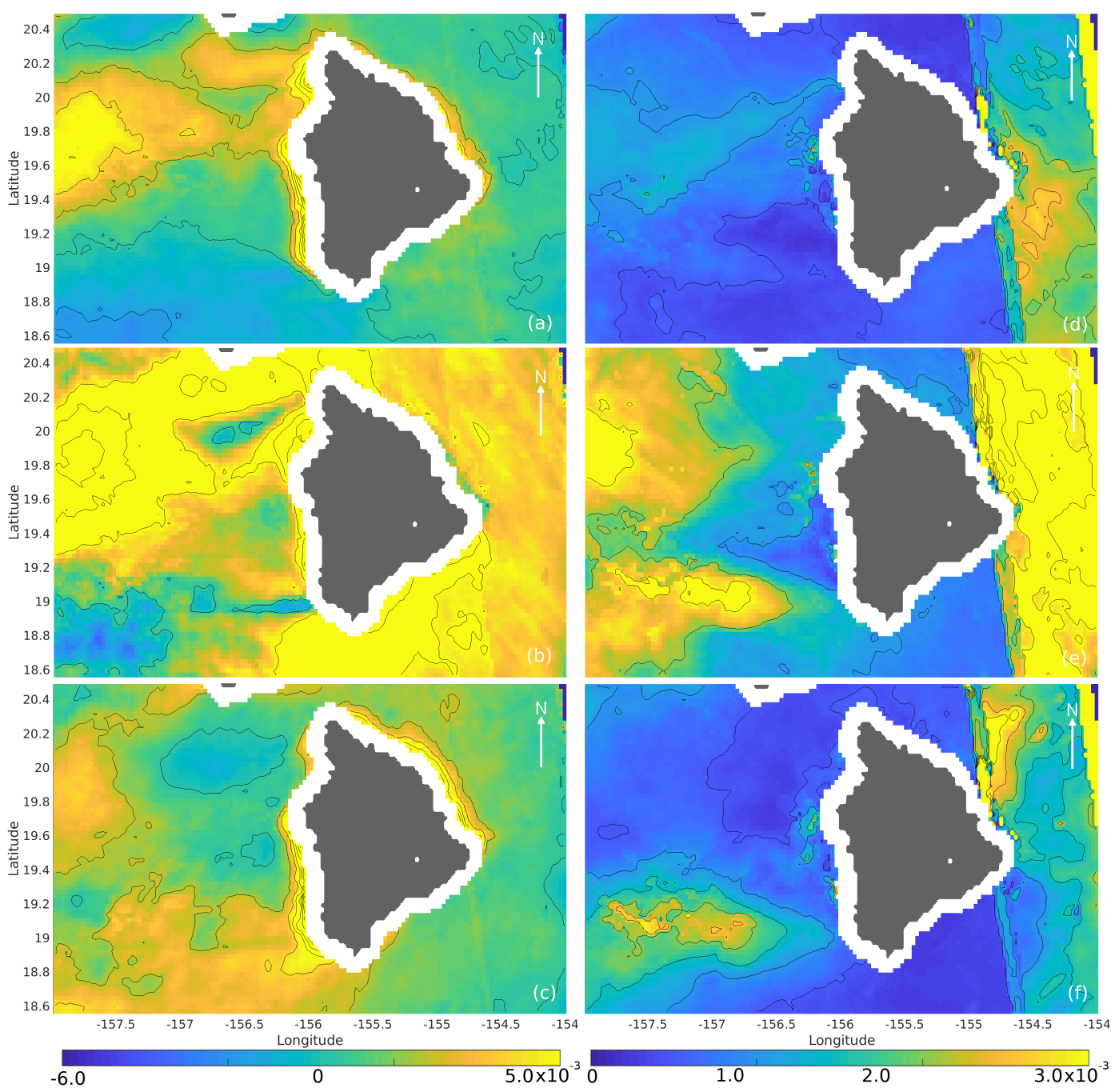

Figure 7. The average difference between linear values of measured ASCAT $\sigma^{0}$ and HRCM predicted $\sigma^{0}$ shown for fore (a), mid (b), and aft (c) beams. Corresponding normalized standard deviation of the difference values are shown to the right in panels $(\mathbf{d}-\mathbf{f})$. The land is shown in gray and the land contamination buffer is shown in white.

Like the QuikSCAT comparison, larger differences in $\sigma^{0}$ can be seen in the high wind speed areas. The effects of azimuth angle can be seen especially when comparing the fore and aft azimuth looks. Immediately west of the island, the average differences and standard deviation values are low for all looks. This is interesting because, like QuikSCAT, ASCAT ambiguity-selected wind directions do not show the reverse flow. The low average difference and low normalized standard deviation values west of the island suggest that ASCAT can be detecting the reverse flow. Imperfect ambiguity selection may be why ASCAT winds do not show this feature. The variance in differences in the high wind speed areas suggest that the orientation of the wind speed tail and placement of vortices by HRCM does not match what ASCAT $\sigma^{0}$ is detecting. 
ASCAT measured $\sigma^{0}$ and NWP predicted $\sigma^{0}$ are compared in Figure 8. ASCAT $\sigma^{0}$ show high differences from NWP $\sigma^{0}$ in the high wind speed regions and low differences immediately west of the island. The variation seen immediately west of the island suggests that ASCAT $\sigma^{0}$ can be detecting something other than the trade wind flow.
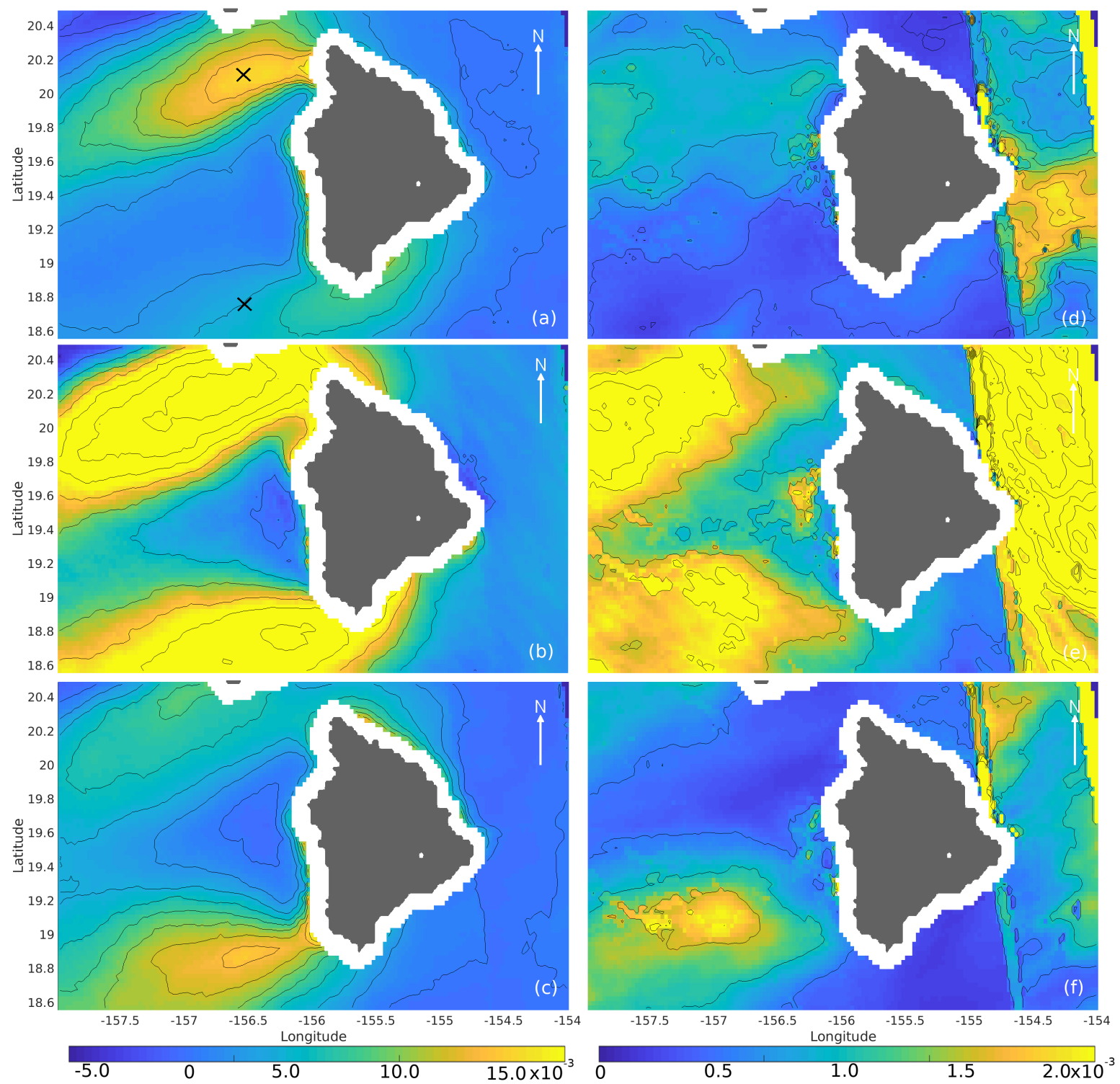

Figure 8. The average difference between linear values of measured ASCAT $\sigma^{0}$ and NWP predicted $\sigma^{0}$ shown for fore (a), mid (b), and aft (c) beams. Corresponding normalized standard deviation of the difference values are shown to the right in panels $(\mathbf{d}-\mathbf{f})$. The land is shown in gray and the land contamination buffer is shown in white. The Xs in (a) indicate where the wind speeds are taken from for the plots in Figure 5.

\subsection{Summary}

Low differences in $\sigma^{0}$ and low standard deviation values compared to HRCM predicted $\sigma^{0}$ immediately west of the island suggest that the scatterometer $\sigma^{0}$ can be detecting the reverse flow. Comparison with the coarse NWP winds supports the idea that UHR $\sigma^{0}$ from both sensors are detecting the island induced perturbation of trade wind flow. Higher difference in $\sigma^{0}$ and standard deviation values in the HRCM comparisons in the high wind speed areas suggest that HRCM is misplacing key 
wind features. The following section details why the ambiguity-selected scatterometer wind fields may not show the reverse flow wind features despite these features possibly being present in the $\sigma^{0}$ field.

\section{Nudging Field and Median Filter Window Size}

The nudging field and median filter-based ambiguity selection scheme have a significant effect on the ambiguity-selected scatterometer wind field. They ensure general wind trends are present, but also smooth the final result. In [15], Kilpatrick et al. identify the nudging field and median filter window size as potential problems for wind retrieval in this area for coarse resolution estimates. In this section, we explore the effects of nudge winds and median filter window size on UHR estimates.

We employ simulation to explore the effects of the nudging field and median filter window size on UHR wind estimation in the lee of the Big Island. Simulated $\sigma^{0}$ measurements are created from fine resolution HRCM winds using the QMOD3 GMF. Monte Carlo noise is added to the $\sigma^{0}$ measurements. The noisy $\sigma^{0}$ measurements are then processed using traditional QuikSCAT UHR processing.

At each UHR WVC, wind retrieval results in two to four ambiguous wind vector solutions. Among the ambiguous solutions are the wind speed and direction values that are close to the true wind model values. Those ambiguities are the desired result of ambiguity selection. We chose to focus on QuikSCAT for this analysis; however, ASCAT yields similar results.

We first examine the effects of the nudging field. Traditional QuikSCAT UHR wind estimation uses L2B wind fields as a nudging fields and L2B is nudged with NWP wind fields. Consequently, UHR is indirectly nudged with coarse $50 \mathrm{~km}$ NWP model winds. Below, we contrast coarse and high resolution nudging fields.

Figure 9 shows a simulated HRCM wind field nudged with an L2B wind field. Where we expect the directions in (a) to show the reverse flow, there are no reverse flow features. Alternatively, choosing the original high resolution HRCM wind field as a nudging field reveals the expected wind features. Comparing (a) and (c) reveals that the ambiguities of the WVCs in lee of the Big Island can represent the reverse flow feature, the difference being the nudging field. Thus, key features can be misrepresented or absent in the ambiguity-selected wind field if the nudging field is low resolution.

After the ambiguous field is nudged, it is processed with the median filter-based ambiguity selection scheme. As mentioned earlier, following the standard approach, a median filter is chosen because it reduces noise and preserves wind fronts. Figure $9 \mathrm{~b}$ is the L2B nudged field that has been median filtered with a $42.5 \mathrm{~km}(17 \times 17$ UHR WVC) window. Figure $9 \mathrm{~d}$ shows the HRCM nudged field median filtered with the same window. The main wind direction features (i.e., trade wind flow and/or reverse flow) in (a) and (c) are preserved in (b) and (d), respectively. The median filtered field in (a) does not show the reverse flow because it was not there to begin with. The filter can only preserve and refine what is already there. The reverse flow in (b) is preserved in (d) after the median filter.

The median filter window size affects how well features are resolved. If the median filter window is large, small features relative to the window can be lost. A window size that is too small can be dominated by noise and choose incorrect ambiguities. Figure 10 shows Figure 9 panels (c) and (d) but in a gray scale wind direction field to better illustrate this point. Figure 10a shows the truth nudged field before filtering. Note the reverse flow in the lee of the Big Island and wind features near the other islands (boxed in red). Figure 10b shows the field after filtering with the same $42.5 \mathrm{~km}$ window. The relatively large reverse flow from (a) is still present in (b), but the smaller features (circled) near the other islands are filtered away. These results confirm that the median filter can preserve the reverse flow feature only if the feature is in the nudging field to begin with and the window size is not too large relative to the feature. 

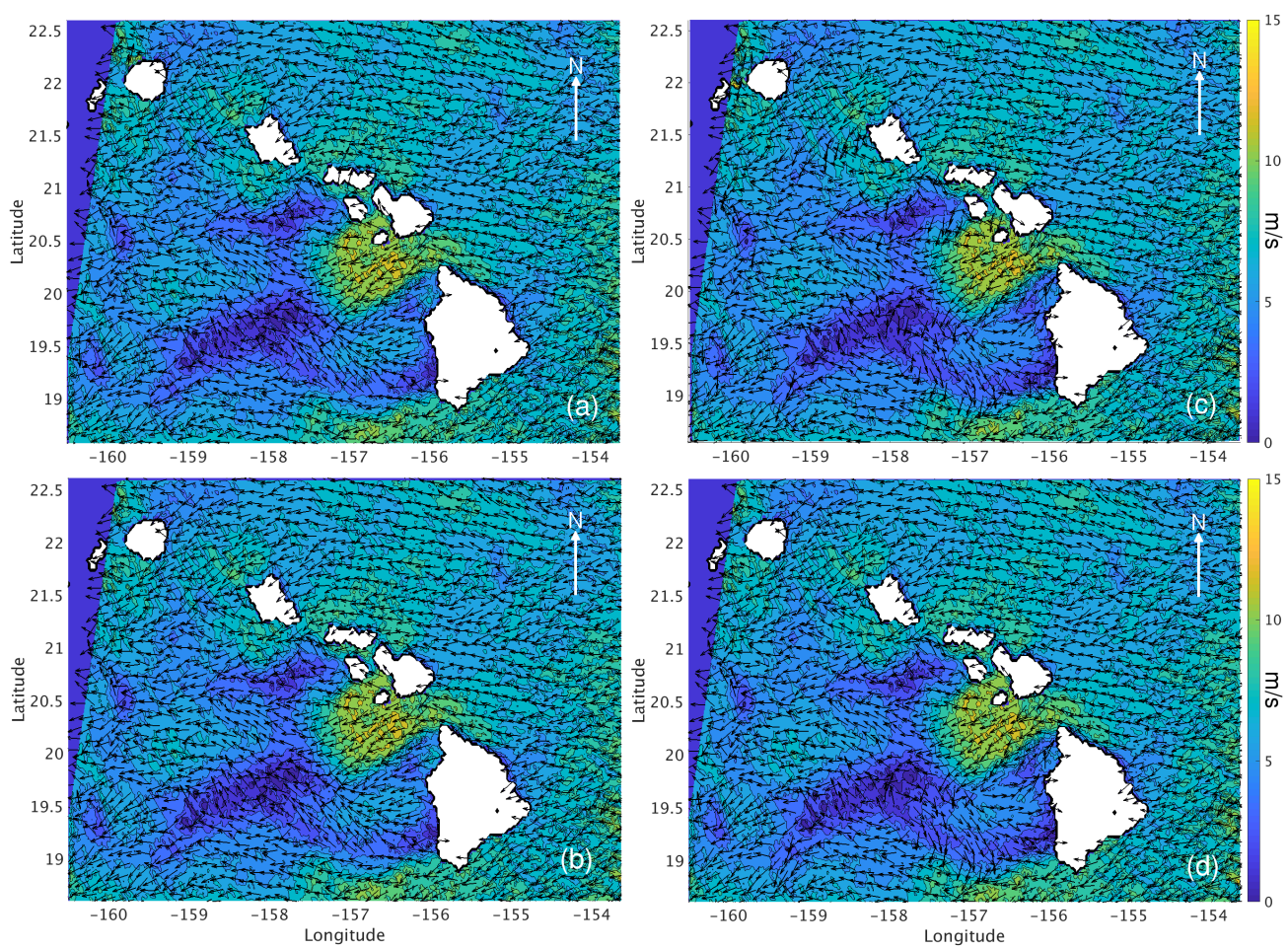

Figure 9. Simulated HRCM wind field nudged with an L2B field (a), simulated field nudged with L2B and median filtered (b), simulated field nudged with the true field (c), simulated field nudged with true field and median filtered (d). The median filter window size is $42.5 \mathrm{~km}$ (17 x 17 UHR WVC). Downsampled wind direction quivers are shown in all panels.

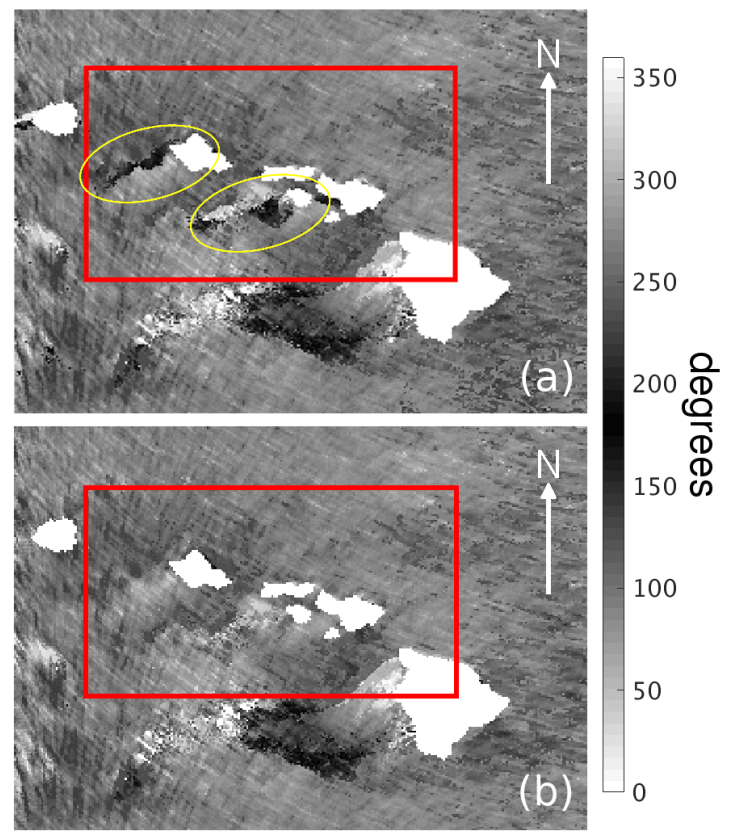

Figure 10. QuikSCAT UHR-derived wind direction fields for different median filter window sizes. (a) shows a swath oriented wind direction field of a simulated HRCM wind field nudged with the true wind field. (b) shows (a) processed with a $42.5 \mathrm{~km}(17 \times 17$ UHR WVC) median filter window. Note how the dark features that differ from the mean flow (circled within the red box) in (a) disappear in (b) after filtering. The land mask is shown in white and the colorbar denotes the wind direction in degrees. 


\section{Discussion and Conclusions}

In the open ocean, QuikSCAT and ASCAT L2B and UHR processing have been proven to provide accurate estimates of ocean wind vectors. The lee of Hawaii's Big Island presents a challenging case for scatterometer wind retrieval due to proximity to land and the fine scale of the expected wind features. In previous work, L2B has been shown to not resolve the complex wind features in this area [15]. This paper shows that selected ambiguity QuikSCAT and ASCAT UHR winds also do not resolve the reverse flow and vortices in the wind fields as predicted by HRCM. However, comparison of scatterometer measured $\sigma^{0}$ to HRCM predicted $\sigma^{0}$ show that the scatterometer $\sigma^{0}$ can be detecting the reverse flow features. The $\sigma^{0}$ analysis also suggests that HRCM winds are inaccurately predicting the locations of key reverse flow features compared to the scatterometer $\sigma^{0}$. It is thought that incorporating high resolution scatterometer data into the HRCM could result in better wind prediction.

The reverse flow features may be missing in the scatterometer ambiguity-selected wind field due to the coarse resolution of the nudging fields and the large window size for the median filter in the UHR ambiguity selection, which causes erroneous ambiguity selection leading to wind direction errors. To improve UHR wind retrieval in this area, a better representation of fine scale features near Hawaii is needed. This can be provided by improved HRCM winds used to nudge the scatterometer ambiguity selection.

Author Contributions: Conceptualization and methodology, N.H. and D.G.L.; validation, T.K.; writing—original draft preparation, N.H.; writing-review and editing, D.G.L. and T.K. All authors have read and agreed to the published version of the manuscript.

Funding: This research was partially funded by NASA Grants No. 80NSSC19K0057 and NNX14AL83Gl.

Acknowledgments: UHR wind products can be provided on request through the Scatterometer Climate Record Pathfinder (SCP) at www.scp.byu.edu.

Conflicts of Interest: The authors declare no conflict of interest.

\section{References}

1. Ulaby, F.T.; Long, D.G. Microwave Radar and Radiometric Remote Sensing; The University of Michigan Press: Ann Arbor, MI, USA, 2014.

2. Long, D.G. Polar Applications of Spaceborne Scatterometers. IEEE J. Sel. Top. Appl. Earth Observ. 2017, 10, 2307-2320. [CrossRef] [PubMed]

3. Lindell, D.B.; Long, D.G. High-Resolution Soil Moisture Retrieval with ASCAT. IEEE Geosci. Remote Sens. Lett. 2016, 13, 972-976. [CrossRef]

4. Lindell, D.; Long, D. Multiyear Arctic ice classification using ASCAT and SSMIS. Remote Sens. 2016, 8, 294. [CrossRef]

5. Chang, P.S.; Jelenak, Z.; Sienkiewicz, J.M.; Knabb, R.; Brennan, M.J.; Long, D.G. Operational use and impact of satellite remotely sensed ocean surface vector winds in the marine warning and forecasting environment. Oceanography 2009, 22, 194-207. [CrossRef]

6. Wentz, F.J.; Ricciardulli, L.; Rodriguez, E.; Stiles, B.W.; Bourassa, M.A.; Long, D.G.; Hoffman, R.N.; Stoffelen, A.; Verhoef, A.; O'Neill, L.W.; et al. Evaluating and extending the ocean wind climate data record. IEEE J. Sel. Top. Appl. Earth Observ. 2017, 10, 2165-2185. [CrossRef] [PubMed]

7. Owen, M.P.; Long, D.G. Land Contamination Compensation for QuikSCAT Near-Coastal Wind Retrieval. IEEE Trans. Geoscie. Remote Sens. 2009, 47, 839-850. [CrossRef]

8. Physical Oceanography Distributed Active Archive Center (PO.DAAC). Seawind's User Guide; Rev. 3.0; Physical Oceanography Distributed Active Archive Center: Pasadena, CA, USA, 2006.

9. Hersbach, H.; Stoffelen, A.; de Haan, S. An improved C-band scatterometer ocean geophysical model function: CMOD5. J. Geophys. Res. Oceans 2007, 112. [CrossRef]

10. Naderi, F.M.; Freilich, M.H.; Long, D.G. Spaceborne radar measurement of wind velocity over the ocean-an overview of the NSCAT scatterometer system. Proc. IEEE 1991, 79, 850-866. [CrossRef]

11. Shaffer, S.J;; Dunbar, R.S.; Hsiao, S.V.; Long, D.G. A median-filter-based ambiguity removal algorithm for NSCAT. IEEE Trans. Geosci. Remote Sens. 1991, 29, 167-174. [CrossRef] 
12. Schultz, H. A circular median filter approach for resolving directional ambiguities in wind fields retrieved from spaceborne scatterometer data. J. Geophys. Res. 1990, 95, 5291-5303. [CrossRef]

13. Xie, S.P.; Liu, W.T.; Liu, Q.; Nonaka, M. Far-reaching effects of the Hawaiian islands on the Pacific Ocean-atmosphere system. Science 2001, 292, 2057-2060. [CrossRef] [PubMed]

14. Sakamoto, T.T.; Sumi, A.; Emori, S.; Nishimura, T.; Hasumi, H.; Suzuki, T.; Kimoto, M. Far-reaching effects of the Hawaiian Islands in the CCSR/NIES/FRCGC high-resolution climate model. Geophys. Res. Lett. 2004, 12. [CrossRef]

15. Kilpatrick, T.; Xie, S.; Tokinaga, H.; Long, D.; Hutchings, N. Systematic scatterometer wind errors near coastal mountains. Earth Space Sci. 2019, 6, 1900-1924. [CrossRef] [PubMed]

16. Nickerson, E.C.; Dias, M.A. On the existence of atmospheric vortices downwind of Hawaii during the HAMEC project. J. Appl. Meteorol. 1981, 20, 868-873. [CrossRef]

17. Smith, R.B.; Grubišić, V. Aerial observations of Hawaii's wake. J. Atmos. Sci. 1993, 50, 3728-3750. [CrossRef]

18. Patzert, W.C. Eddies in Hawaiian Waters; Technical report; Hawaii Inst of Geophysics: Honolulu, HI, USA, 1969.

19. Zhang, C.; Yuqing Wang, A.L.; Hamilton, K. Configuration and evaluation of the WRF model for the study of Hawaiian regional climate. Mon. Weather Rev. 2012, 140, 3259-3277. [CrossRef]

20. Yang, J.; Zhang, J. Evaluation of ISS-RapidScat wind vectors using buoys and ASCAT data. Remote Sens. 2018, 10, 648. [CrossRef]

21. Asia-Pacific Data Research Center. 2019. Available online: http://apdrc.soest.hawaii.edu/ (accessed on 30 January 2020).

22. Zhang, C.; Wang, Y.; Hamilton, K.; Lauer, A. Dynamical downscaling of the climate for the Hawaiian Islands. Part I: Present day. J. Clim. 2016, 29, 3027-3048. [CrossRef]

23. Lindsley, R.; Long, D.G. Enhanced-Resolution Reconstruction of ASCAT Backscatter Measurements. IEEE Trans. Geosci. Remote Sens. 2016, 54, 2589-2601. [CrossRef]

24. Williams, B.A.; Long, D.G. A reconstruction approach to scatterometer wind vector field retrieval. IEEE Trans. Geosci. Remote Sens. 2011, 49, 1850-1864. [CrossRef]

25. Plagge, A.; Vandemark, D.C.; Long, D.G. Coastal validation of ultra-high resolution wind vector retrieval from QuikSCAT in the Gulf of Maine. IEEE Geosci. Remote Sens. Lett. 2009, 6, 413-417. [CrossRef]

26. Williams, B.A.; Owen, M.P.; Long, D.G. The ultra high resolution QuikSCAT product. In Proceedings of the 2009 IEEE Radar Conference, Pasadena, CA, USA, 4-8 May 2009; pp. 1-6. [CrossRef]

27. Williams, B.A.; Long, D.G. Estimation of Hurricane Winds from SeaWinds at Ultra High Resolution. IEEE Trans. Geosci. Remote Sens. 2008, 46, 2924-2935. [CrossRef]

28. Owen, M.P.; Long, D.G. Simultaneous Wind and Rain Estimation for QuikSCAT at Ultra-High Resolution. IEEE Trans. Geosci. Remote Sens. 2011, 49, 1865-1878. [CrossRef]

29. Owen, M.P.; Long, D.G. Prior Selection for QuikSCAT Ultra-High Resolution Wind and Rain Retrieval. IEEE Trans. Geosci. Remote Sens. 2013, 51, 1555-1567. [CrossRef]

30. Hutchings, N.; Long, D.G. Improved Ultrahigh-Resolution Wind Retrieval for RapidScat. IEEE Trans. Geosci. Remote Sens. 2018, 57, 3370-3379. [CrossRef]

31. Lindsley, R.; Blodgett, J.R.; Long, D.G. Analysis and Validation of High-Resolution Wind from ASCAT. IEEE Trans. Geosci. Remote Sens. 2016, 54, 5699-5711. [CrossRef]

32. Lindsley, R.D. Enhanced-Resolution Processing and Applications of the ASCAT Scatterometer. Ph.D. Thesis, Brigham Young University, Provo, UT, USA, 2015.

33. Freilich, M.H.; Dunbar, R.S. The accuracy of the NSCAT 1 vector winds: comparisons with National Data Buoy Center buoys. J. Geophys. Res. 1999, 104, 11232-11246. [CrossRef]

34. Liu, W.T.; Tang, W. Equivalent Neutral Wind; Jet Propulsion Laboratory Publication: Pasadena, CA, USA, 1996; pp. 96-117.

(C) 2020 by the authors. Licensee MDPI, Basel, Switzerland. This article is an open access article distributed under the terms and conditions of the Creative Commons Attribution (CC BY) license (http:/ / creativecommons.org/licenses/by/4.0/). 